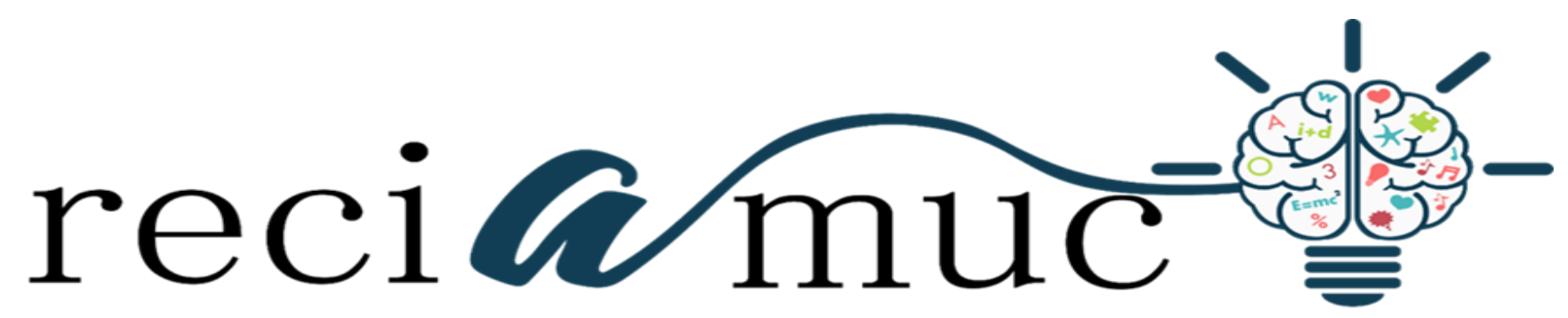

Revista científica de investigación actualización del mundo de las ciencias

$$
\begin{aligned}
& \text { David Ricardo Silva Guachilema a }{ }^{\text {a }} \text { Juan Carlos Salazar }{ }^{\text {b; }} \\
& \text { Johnny Correa Michilena }^{\text {c; }} \text { Javier Stalin Villagómez Toral }{ }^{\text {d }}
\end{aligned}
$$

Importancia de las troponinas en el diagnóstico de infarto agudo de miocardio (IAM)

Importance of troponins in the diagnosis of acute myocardial infarction (AMI)

Revista Científica de Investigación actualización del mundo de las Ciencias. Vol. 3 núm., 4, octubre: 2588-0748, 2019, pp. 22-40

DOI: 10.26820/reciamuc/3.(4).octubre.2019.22-40

URL: http://reciamuc.com/index.php/RECIAMUC/article/view/386

Código UNESCO: 3205 Medicina Interna

Tipo de Investigación: Artículo de Revisión

(C) RECIAMUC; Editorial Saberes del Conocimiento, 2019

Recibido: 15/05/2019

Aceptado: 07/08/2019

Publicado: 01/10/2019

Correspondencia: daricsilvag@hotmail.com

a. Médico; Investigador Independiente; Guayaquil, Ecuador, daricsilvag@ hotmail.com

b. Médico; Investigador Independiente; Guayaquil, Ecuador, juank8sf@yahoo.es

c. Médico; Investigador Independiente; Guayaquil, Ecuador, johnnycorreamichilena @ outlook.com

d. Médico; Investigador Independiente; Guayaquil, Ecuador, javime8590@ outlook.com 


\section{Importancia de las troponinas en el diagnóstico de infarto agudo de miocardio}

(IAM)

Vol. 3, núm. 4., (2019)

David Ricardo Silva Guachilema; Juan Carlos Salazar; Johnny Correa Michilena; Javier Stalin

Villagómez Toral

\section{RESUMEN}

El uso de la troponina cardiaca (cTn) como el biomarcador para la evaluación de los pacientes con posible diagnóstico de infarto agudo de miocardio (IAM/IM) se ha recomendado desde hace ya casi veinte años. El correcto diagnostico o descarte del IAM aún se ha considerado un verdadero desafío al que se enfrentan los especialistas médicos en sus respectivas unidades de servicios de atención de urgencias. En clínica, se ha evidenciado la mejoría en el diagnóstico del IAM por el uso de este biomarcador, toda vez que sus valores de concentración han sido interpretados adecuadamente en el contexto de la historia clínica del paciente, principalmente. Es por ello que ha surgido el interés en desarrollar el presente trabajo investigativo en el marco de un diseño bibliográfico y bajo una metodología de revisión sistemática, en la que el objetivo planteado ha sido extraer de la literatura científico académica reciente cual vendría siendo la importancia de las troponinas en el diagnóstico del infarto agudo de miocardio (IAM/IM), siendo necesario para tales fines aprovechar las más reconocidas bases de datos, de las que se pretende igualmente encontrar aquellos aspectos que, en síntesis, denotan esta complicación, su etiología, sintomatología y tipología. Los resultados encontrados demuestran que efectivamente la literatura respecto a esta temática se encuentra bastante documentada y actualizada, por lo que no resultó difícil exponer sobre los aspectos antes mencionados. Las conclusiones estriban en torno a la reconocida importancia que más que todo en el ámbito clínico se le ha dado a las troponinas para el certero diagnóstico del IAM.

Palabras claves: Biomarcador; Especialistas; Urgencias; Clínica; Etiología. 


\title{
Importancia de las troponinas en el diagnóstico de infarto agudo de miocardio (IAM)
}

Vol. 3, núm. 4., (2019)

David Ricardo Silva Guachilema; Juan Carlos Salazar; Johnny Correa Michilena; Javier Stalin Villagómez Toral

\begin{abstract}
The use of cardiac troponin (cTn) as the biomarker for the evaluation of patients with a possible diagnosis of acute myocardial infarction (AMI / MI) has been recommended for almost twenty years. The correct diagnosis or discard of AMI has still been considered a real challenge faced by medical specialists in their respective emergency care units. In clinical practice, the improvement in the diagnosis of AMI has been evidenced by the use of this biomarker, since its concentration values have been interpreted properly in the context of the patient's clinical history, mainly. That is why the interest in developing this research work has arisen within the framework of a bibliographic design and under a systematic review methodology, in which the objective has been to extract from recent scientific-academic literature what would be the importance of troponins in the diagnosis of acute myocardial infarction (AMI / MI), being necessary for such purposes to take advantage of the most recognized databases, which are also intended to find those aspects that, in synthesis, denote this complication, its etiology, symptomatology and typology The results found show that indeed the literature regarding this subject is fairly documented and updated, so it was not difficult to present the above-mentioned aspects. The conclusions are based on the recognized importance that, above all, in the clinical setting has been given to troponins for the accurate diagnosis of AMI.
\end{abstract}

Key words: Biomarker; Specialists; Emergencies; Clinic; Etiology. 


\section{Importancia de las troponinas en el diagnóstico de infarto agudo de miocardio}

Vol. 3, núm. 4., (2019)

David Ricardo Silva Guachilema; Juan Carlos Salazar; Johnny Correa Michilena; Javier Stalin

Villagómez Toral

\section{Introducción.}

El infarto agudo de miocardio (IAM/IM) se trata de "un episodio de necrosis miocárdica causado por un síndrome isquémico inestable”, siendo en base a la clínica, el electrocardiograma (ECG), los análisis complementarios, los estudios por imágenes invasivos y no invasivos y la anatomía patológica que se diagnostica y evalúa. Así miso la cardiopatía isquémica la que a nivel mundial principalmente contribuye a la carga de enfermedad y paralelamente, "la carga global de enfermedad cardiovascular e infarto agudo de miocardio se desplazó a los países de ingresos de bajos a medios, donde se producen actualmente más del $80 \%$ de las muertes por enfermedad cardiovascular”. (Anderson \& Morrow, 2017)

Recientes datos aportados por la Organización Mundial de la Salud [OMS] (2019) refieren que "las enfermedades cardiovasculares (ECV) son un conjunto de trastornos del corazón y de los vasos sanguíneos" que representan la causa principal de defunción en todo el mundo, matando personas con una marcada diferencia (más del 80\%) en los países de bajos y medianos ingresos. "El consumo de tabaco, una dieta malsana y la inactividad física aumentan el riesgo de infartos de miocardio" (OMS, 2019)

Vitón, Lorenzo, Linares, Lazo, \& Godoy (2018) señala que el infarto agudo de miocardio (IMA) es uno de los dos formatos clínicos más frecuentes en que se deriva la cardiopatía isquémica (CI). Este autor afirma que este tipo de complicaciones provocaban, a principios del siglo XX, menos del $10 \%$ de todas las muertes en el mundo, dato que contrasta con el presente, pues ahora representan casi el 50\% de los decesos en los países desarrollados, y el $25 \%$ en los países en vías 


\section{Importancia de las troponinas en el diagnóstico de infarto agudo de miocardio (IAM)}

Vol. 3, núm. 4., (2019)

David Ricardo Silva Guachilema; Juan Carlos Salazar; Johnny Correa Michilena; Javier Stalin Villagómez Toral

de desarrollo. Indicó que la OMS, pronostica que la enfermedad isquémica del corazón será responsable de 11,1 millones de muertes para el 2020. (párr. 3-4)

En base a los antes dicho, bien pudiera deducirse lo trascendente que es conocer sobre esta enfermedad y todos los aspectos fundamentales asociados a ella, y tal viene siendo el caso aquí planteado, ya que múltiples factores han despertado el interés por conocer la importancia de las troponinas en el diagnóstico de esta condición.

Es por ello que, en lo sucesivo, el objetivo será, no solamente exponer sobre aquellos aspectos necesarios para comprender lo que implica el infarto agudo de miocardio (IAM), sino más específicamente el de extraer de la literatura científico académica vigente cuál es la importancia de las troponinas en su diagnóstico.

\section{Materiales y Métodos}

Las herramientas y materiales de trabajo que se conciben para el desarrollo de esta investigación son los siguientes: computadores personales con conexión a internet y un cúmulo de contenidos científico-académicos diversos, con los que se persigue aportar información de calidad y actualizada, respecto la importancia de las troponinas en el diagnóstico de IAM. Se escoge un diseño bibliográfico, bajo una metodología de revisión sistemática.

A junio de 2018, se realizó una búsqueda estructurada de la literatura en las bases de datos EBSCO, ScienceDirect, PubMed, LILACS, Embase, SciELO y Cochrane Library con los términos en castellano "infarto agudo miocardio", "diagnóstico infarto agudo miocardio", "troponinas en el 


\section{Importancia de las troponinas en el diagnóstico de infarto agudo de miocardio}

(IAM)

Vol. 3, núm. 4., (2019)

David Ricardo Silva Guachilema; Juan Carlos Salazar; Johnny Correa Michilena; Javier Stalin

Villagómez Toral

diagnóstico de infarto" e "infarto agudo miocardio + troponinas"; y en inglés, "acute myocardial infarction" y "myocardial infarction", obteniéndose como resultados de búsqueda, de manera conjunta; es decir, sumando el total los títulos mostrados en referencia a registros bibliográficos en cada base de datos, un total general acumulado de más de un centenar de miles de fuentes relacionadas con los términos usados.

En función de determinar la importancia de las troponinas en el diagnóstico de IAM, se aplicaron filtros a dichos resultados de búsquedas, lo que gradualmente fue arrojando nuevos resultados, en la medida en que se iban aplicando. De entre los múltiples filtros preestablecidos en cada interfaz de búsqueda de las bases de datos, fundamentalmente se escogieron los que se asociaban al tipo de fuente bibliográfica, año de publicación, idioma y género; lo que en concreto facilitó la escogencia de solamente aquellos trabajos relevantes por su correlación temática e identificados como: artículos científicos originales y de revisión, guías de manejo, revisiones sistemáticas con o sin meta análisis, artículos de revisión narrativa, estudios de cohorte, ensayos clínicos, protocolos, ebooks, boletines y folletos de reconocidas organizaciones internacionales y nacionales, tesis de posgrado y doctorado, noticias científicas y otros documentos e información de interés científico académico publicados en los últimos 10 años, contados a partir del presente año, es decir, de 2009 al 2019. Se desestimaron estudios de casos y controles; series y reportes de casos; editoriales, cartas al editor y otros tipos de publicaciones de poca relevancia y escaso valor científico académico. 


\section{Importancia de las troponinas en el diagnóstico de infarto agudo de miocardio (IAM)}

Vol. 3, núm. 4., (2019)

David Ricardo Silva Guachilema; Juan Carlos Salazar; Johnny Correa Michilena; Javier Stalin Villagómez Toral

Seguidamente, se efectúa la selección y clasificación de la literatura científico académica físicamente disponible, aplicando, en términos generales, los criterios antes mencionados, siendo a partir de allí que el equipo investigador le da la correspondiente lectura crítica y análisis de toda esa evidencia científica, lo que resultó consecutiva y consensuadamente en el fundamento de las ideas y planteamientos aquí plasmados.

\section{Resultados}

De Pérez (2019) se comprende que, es impreciso que frecuentemente se asuma que el IAM es sinónimo de infarto, ya que en realidad, éste último se refiere a una concepción más general, pues se trata de la muerte celular (necrosis) de todo o parte de un órgano cualquiera por falta de irrigación sanguínea, debiéndose tal insuficiencia de sangre a una obstrucción total o parcial (aterosclerosis: endurecimiento o estenosis: estrechez) de la arteria correspondiente; mientras que el IAM igualmente se debe a un insuficiente riego sanguíneo debido a la obstrucción de una arteria pero específicamente del músculo cardiaco, he allí su particularidad.

Desde otra perspectiva, la Sociedad Española de Medicina Interna [SEMI] (2019) expone que el infarto consiste en la obstrucción brusca del paso de sangre a lo largo de una arteria coronaria con la subsiguiente muerte de la parte del corazón que es irrigada (alimentada) por dicha arteria, y por ello esta condición es particularmente considerada de urgencia, asociada a una elevada mortalidad. Continúan detallando que se debe considerar que existen dos tipos de infarto de miocardio, que son: con onda Q (o síndrome coronario agudo con elevación del segmento ST), que se produce por la obstrucción prolongada de una de las arterias coronarias importantes, lo que 


\section{Importancia de las troponinas en el diagnóstico de infarto agudo de miocardio}

(IAM)

Vol. 3, núm. 4., (2019)

David Ricardo Silva Guachilema; Juan Carlos Salazar; Johnny Correa Michilena; Javier Stalin

Villagómez Toral

lleva a la muerte de una zona más o menos grande del corazón; y, sin onda Q, en el que la falta de irrigación generalmente afecta a arterias del corazón un poco más pequeñas, haciendo posible que no muera una zona tan extensa del corazón y a su vez favorezca un mejor pronóstico. El infarto de miocardio sin onda $\mathrm{Q}$ se agrupa junto a la angina inestable, dentro de los síndromes coronarios agudos sin elevación del segmento ST. Respecto a ambas definiciones, cabe aclarar que la "onda Q" está referida a una pequeña cicatriz que puede observarse en el electrocardiograma una vez que ha pasado la fase aguda del infarto. (SEMI, 2019)

El Hospital Universitario Austral (2019) refiere que esta condición, que también es comúnmente conocida como ataque cardíaco, se produce por una exigua irrigación sanguínea al corazón, y por ende, falta de oxígeno. Ésa situación es llamada: isquemia, que puede originarse por varias razones, entre las que destacan: a) engrosamiento de las paredes de las arterias coronarias, debido una progresiva acumulación de grasa (placa), que a su vez es a causa del colesterol, y que es posible que pueda llegar al punto taparse; b) por un coágulo, ocurrido en otra parte del organismo, que bloquea una arteria coronaria (a veces por el engrosamiento de placa), que impide llevar sangre al corazón; c) sangramiento y formación coágulo en el interior de la arteria, que ocurre a raíz del rompimiento de la placa; y d) por espasmo (de los que aún se desconocen sus causas) en una arteria coronaria, entendido como una contracción que dificulta o interrumpe el flujo sanguíneo. Evidentemente, las células cardíacas mueren si el flujo sanguíneo se bloquea y el órgano no recibe sangre ni oxígeno.

Wayne (2016) aporta significativos detalles sobre esta condición que bien valen la pena destacar. El autor expone que el infarto agudo de miocardio (IAM/IM) “es una necrosis miocárdica 


\section{Importancia de las troponinas en el diagnóstico de infarto agudo de miocardio (IAM)}

Vol. 3, núm. 4., (2019)

David Ricardo Silva Guachilema; Juan Carlos Salazar; Johnny Correa Michilena; Javier Stalin Villagómez Toral

que se produce como resultado de la obstrucción aguda de una arteria coronaria”. El IM agudo, junto con la angina inestable, “componen un síndrome coronario agudo (...) que incluye tanto el tipo sin elevación del segmento ST (IMSEST) y con elevación del segmento ST (IMEST)”. En términos fisiopatológicos, coincide con otros tratadistas al referir que "El infarto de miocardio se define como la necrosis miocárdica en un entorno clínico compatible con isquemia miocárdica" (párr. 1,3-4)

\section{Definición universal de infarto de miocardio}

Bazzino (2013) expone que en el año 2000 nació el documento que redefinió el concepto infarto de miocardio (IM) y mediante el mismo se logró un consenso general de que dicho término se usaría en base a la consideración de atributos tales como:

[...] el monto de la pérdida de miocitos (tamaño del infarto), las circunstancias en las que se presenta (espontáneo o en el contexto de una intervención coronaria) y el tiempo de evolución de la necrosis en relación con el momento de la observación. (p. 403)

Continua el tratadista exponiendo que, luego, en 2007, se destacan otros dos aspectos fundamentales para presentar una nueva y universal definición de IM, que por una parte enfatizaba la importancia de la troponina en el diagnóstico, y, por la otra, definía cinco tipos de infarto (que más adelante se mencionarán). Posteriormente, en 2012, debido a la necesidad de actualizar la anterior definición universal por las limitaciones propias que se evidenciaban en su conceptualización, y al desarrollo de herramientas diagnósticas y el mejor entendimiento del valor, fue que surgió la tercera definición universal de IM en la que se destacan como aspectos centrales: 


\section{Importancia de las troponinas en el diagnóstico de infarto agudo de miocardio}

(IAM)

Vol. 3, núm. 4., (2019)

David Ricardo Silva Guachilema; Juan Carlos Salazar; Johnny Correa Michilena; Javier Stalin

Villagómez Toral

“[...] los síntomas clínicos, los biomarcadores cardíacos y las alteraciones electrocardiográficas compatibles con isquemia miocárdica". (p. 403-404)

Por lo tanto, alega el experto, es así como con la tercera definición universal se hace énfasis en:

- El importante significado que tiene la elaboración de la historia clínica de síntomas isquémicos en un lapso de no menos de 20 minutos, no necesariamente fundados en el típico dolor torácico.

- El análisis de los escenarios clínicos en los que ocurre el IM.

- El papel preponderante que, primeramente, siguen representando los biomarcadores en el diagnóstico, y segundo, la mejor ponderación que le es reconocida a las pruebas con imágenes.

Tipos de IAM

Calvachi et al. (2017) cita a (Jneid, Alam, Virani \& Bozkur; 2013) para indicar que la Organización Mundial de la Salud, con fines diagnósticos, epidemiológicos e investigativos, desde 1971 ha procurado definir mejor esta entidad. Continúa diciendo, en base a (White, Thygesen, Alpert \& Jaffe; 2014), que ha sido en razón de lo antes indicado que, a lo largo de este tiempo, se han originado diversas clasificaciones, y una de esas es la tercera definición de infarto, no obstante, en una previa investigación, Thygesen et al. (2013) clasificaron el IAM de la siguiente manera:

- Tipo 1: infarto agudo de miocardio espontáneo. 


\section{Importancia de las troponinas en el diagnóstico de infarto agudo de miocardio (IAM)}

Vol. 3, núm. 4., (2019)

David Ricardo Silva Guachilema; Juan Carlos Salazar; Johnny Correa Michilena; Javier Stalin Villagómez Toral

- Tipo 2: infarto agudo de miocardio secundario a desequilibrio isquémico.

- Tipo 3: infarto agudo de miocardio que conduce a muerte cuando aún no se dispone de los resultados de biomarcadores.

- Tipo 4 A: infarto agudo de miocardio relacionado con intervención coronaria percutánea.

- Tipo 4 B: infarto agudo de miocardio relacionado con trombosis del stent.

- Tipo 5: infarto agudo de miocardio relacionado con derivación aorto-coronaria con injerto.

(Calvachi et al.; 2017; p. 593)

Criterios para el diagóstico de infarto agudo de miocardio

Bazzino (2013) indica que "[...] el término infarto agudo de miocardio (IAM) debe ser utilizado cuando existe evidencia de necrosis miocárdica en un escenario clínico consistente con isquemia miocárdica aguda". Es por ello que, continua el experto, en base a tales condiciones que cualquier criterio que se menciona a continuación aplicarán para un diagnóstico de IAM:

1. Detección de un incremento y/o caída de los niveles de biomarcadores cardíacos (preferentemente troponinas), con al menos un valor por encima del percentil 99 del límite superior de referencia, y, al menos, una de las siguientes condiciones

a) Síntomas de isquemia.

b) Cambios nuevos, o presumiblemente nuevos, en el segmento ST y/o onda T; o bloqueo de rama izquierda nuevo.

c) Desarrollo de ondas Q patológicas. 


\section{Importancia de las troponinas en el diagnóstico de infarto agudo de miocardio}

(IAM)

Vol. 3, núm. 4., (2019)

David Ricardo Silva Guachilema; Juan Carlos Salazar; Johnny Correa Michilena; Javier Stalin

Villagómez Toral

d) Evidencia imagenológica de una pérdida nueva de miocardio viable, o de una anormalidad nueva en la contractilidad parietal regional.

e) Identificación de trombo intracoronario mediante angiografía o autopsia.

2. Muerte cardíaca con síntomas sugestivos de isquemia miocárdica y alteraciones electrocardiográficas isquémicas o bloqueo de rama izquierda nuevo. La muerte ocurre antes de la dosificación de biomarcadores o antes que los valores de estos alcancen niveles anormales.

3. El infarto de miocardio relacionado con una intervención coronaria percutánea es arbitrariamente definido como una elevación de troponinas cinco veces el valor del percentil 99 del nivel de referencia, o > 20\% si los niveles basales están elevados (estables o en descenso). Adicionalmente se requiere alguna de las siguientes condiciones: a) síntomas sugestivos de isquemia miocárdica; b) nuevos cambios electrocardiográficos isquémicos; c) hallazgos angiográficos consistentes con una complicación del procedimiento, o d) imágenes que evidencien una pérdida nueva de miocardio viable o alteraciones sectoriales nuevas de la contractilidad.

4. Trombosis del stent relacionado a infarto de miocardio, detectado por angiografía o autopsia en el con texto de isquemia miocárdica, y con un ascenso y/o descenso de los niveles de biomarcadores cardíacos, con al menos un valor por encima del percentil 99 del rango de referencia.

5. El infarto de miocardio relacionado con cirugía de revascularización miocárdica es arbitrariamente definido como una elevación superior a diez veces el percentil 99 del rango 


\section{Importancia de las troponinas en el diagnóstico de infarto agudo de miocardio (IAM)}

Vol. 3, núm. 4., (2019)

David Ricardo Silva Guachilema; Juan Carlos Salazar; Johnny Correa Michilena; Javier Stalin Villagómez Toral

de referencia en pacientes con niveles basales normales, y con al menos una de las siguientes condiciones: a) ondas $\mathrm{Q}$ patológicas o bloqueo de rama izquierda nuevo; b) nueva oclusión de puente o arteria nativa documentada angiográficamente, o c) imágenes que evidencien una pérdida nueva de miocardio viable o alteraciones sectoriales nuevas de la contractilidad. (Bazzino; 2013; p. 405)

Examen (prueba) de troponina.

Es un examen que mide los niveles de las proteínas troponina $\mathrm{T}$ y troponina I en la sangre. Estas proteínas (también conocidas con los nombres Troponina I; TnI; Troponina T; TnT; Troponina I cardio-específica; Troponina T cardio-específica; Tnlc; TnTc) se liberan cuando el miocardio sufre algún daño, tal y como ocurre con un ataque cardíaco. Existe una relación proporcional entre la cantidad de troponina T e I en sangre y el daño que se produzca en el corazón. Comúnmente, este examen es aplicado para verificar si se ha presentado un ataque al corazón, cuando el paciente presenta dolor torácico y otros signos de un ataque cardíaco, pudiendo repetirse hasta dos veces más entre las siguientes 6 a 24 horas.

Igualmente, aplicara para aquellos pacientes que presenten dolor torácico; que se cree resulta de una parte del corazón que no está recibiendo suficiente flujo de sangre (angina), que está en desmejora y sin ningún otro signo de un ataque cardíaco.

"La prueba de troponina también se puede hacer para ayudar a detectar y evaluar otras causas de lesión al corazón.” (A.D.A.M. - Enciclopedia Médica, 2017) 


\section{Importancia de las troponinas en el diagnóstico de infarto agudo de miocardio}

Vol. 3, núm. 4., (2019)

David Ricardo Silva Guachilema; Juan Carlos Salazar; Johnny Correa Michilena; Javier Stalin

Villagómez Toral

\section{Diagnóstico, biomarcadores y su importancia}

La Clínica Universidad de Navarra (2019 a) ha publicado que:

Para diagnosticar y realizar el seguimiento del IAM existen diversos protocolos. Como norma general, se diagnostica de IAM si se detecta dolor a nivel del corazón o alteraciones en el electrocardiograma y, además, se produce una elevación de los enzimas cardiacos en sangre como consecuencia de la ruptura celular. Si estos enzimas no aumentan, la lesión puede considerarse reversible.

De la misma manera detallan que el IAM es reconocible por la repentina aparición de unos síntomas característicos, tales como: dolor intenso en el pecho (zona precordial) con una duración de 5 o más minutos, pudiendo este extenderse hacia el brazo izquierdo, hacia la mandíbula, hacia el hombro, hacia la espalda o hacia cuello; además de sensación de malestar general, mareo, náuseas y sudoración. Amplían su explicación describiendo que:

Con un electrocardiograma se demuestran alteraciones evolutivas típicas, y con analítica se valora la elevación de los niveles en sangre de las enzimas cardíacas. Se debe realizar también una radiografía de tórax para ver si el corazón esta agrandado o si hay líquido en los pulmones. (Clinica Universidad de Navarra, 2019 b)

Bazzino (2013) se apoya en el criterio de varios expertos para explicar que la injuria miocárdica se puede diagnosticar mediante la evidencia de una elevación de los niveles de determinados biomarcadores sensibles y específicos, tales como las troponinas o la fracción MB 


\section{Importancia de las troponinas en el diagnóstico de infarto agudo de miocardio (IAM)}

Vol. 3, núm. 4., (2019)

David Ricardo Silva Guachilema; Juan Carlos Salazar; Johnny Correa Michilena; Javier Stalin Villagómez Toral

de la creatinfosfoquinasa (CK). A nivel del corazón, es exclusiva la manifestación de las troponinas

T e I, debido a que son parte del aparato contráctil de los miocitos. Continúa aclarando el hecho de que, no obstante, se identifiquen elevados niveles de estos biomarcadores que indiscutiblemente significan un daño en el músculo cardiaco, conducente a necrosis miocárdica, no debe suponerse cuál ha sido mecanismo subyacente, pues es muy diverso.

Se han propuesto varias explicaciones para la liberación de estas proteínas estructurales desde los miocitos: recambio normal de los miocitos, apoptosis, liberación celular de los productos de degradación de troponina, aumento de la permeabilidad de la pared celular, formación y liberación de vesículas membranosas y necrosis de los miocitos [...] Los biomarcadores preferidos son las troponinas ( $\mathrm{T}$ o I) debido a su alta especificidad por el tejido miocárdico, así como por su alta sensibilidad clínica. La detección de un ascenso y/o descenso de sus niveles séricos resulta imprescindible para el diagnóstico de IAM (Bazzino; 2013; p. 406)

Wayne (2016), de la misma manera expone sobre los marcadores cardiacos, y al respecto ilustra que se refieren a marcadores séricos de la lesión celular miocárdica, y a su vez, son aquellas enzimas cardíacas (CK-MB) y contenidos celulares (troponina I, troponina $\mathrm{T}$, mioglobina) que se liberan a la corriente sanguínea tras la necrosis de las células miocárdicas. Dichos marcadores aparecen después de la lesión, en diferentes períodos, y su concentración disminuye a velocidades disímiles. Actualmente, los marcadores con preferencias de elección son las troponinas (cTn), por representar mayor la sensibilidad y especificidad, sin embargo, estas mismas variables son significativamente distintas entre el grupo de marcadores para detectar la lesión celular miocárdica. 


\section{Importancia de las troponinas en el diagnóstico de infarto agudo de miocardio}

(IAM)

Vol. 3, núm. 4., (2019)

David Ricardo Silva Guachilema; Juan Carlos Salazar; Johnny Correa Michilena; Javier Stalin

Villagómez Toral

Este autor informa que se han comenzado a usar, recientemente, varios ensayos nuevos de alta sensibilidad para troponina cardíaca (hs-cTn), que de la misma manera han sido muy precisos, ya que, en éstos, de manera confiable, se ha demostrado mediciones de niveles de Tn (T o I) tan bajos como 0,003 a 0,006 ng/mL (3 a 6 pg/mL); incluso en otros ensayos de investigación han detectado niveles tan infimos como 0,001 ng/mL (1 pg/mL). En definitiva, el experto refiere que;

[...]las nuevas pruebas de hs-cTn pueden detectar pequeñas cantidades de troponina en muchas personas sanas. Por lo tanto, los niveles de hs-cTn necesitan relacionarse con el rango normal, y se definen como "elevados" solo cuando son más altos que los del 99\% de la población de referencia. Por otra parte, a pesar de que un nivel elevado de troponina indica lesión celular del miocardio, no señala la causa del daño (aunque cualquier elevación de la troponina aumenta el riesgo de resultados adversos en muchos trastornos). Además del síndrome coronario agudo (SCA), muchos otros trastornos cardíacos y no cardíacos pueden elevar los niveles de hs-cTn; no todos los niveles elevados de hs-Tn representan un IM, y no todas las necrosis miocárdicas son el resultado de un síndrome coronario agudo, incluso cuando la etiología es isquémica. Sin embargo, mediante la detección de los niveles más bajos de Tn, los ensayos de hs-cTn permiten la identificación de un IM más temprano que otros ensayos, y han sustituido a otras pruebas de marcadores cardíacos en muchos centros. (Wayne, 2016)

Finalmente, pudiera referirse que la importancia de las troponinas en el diagnóstico de IAM a nivel clínico radica en el hecho de que; en base a la aplicación de un método de medición Tnc de alta sensibilidad que resulte en valores normales en muestras seriadas: 


\section{Importancia de las troponinas en el diagnóstico de infarto agudo de miocardio (IAM)}

Vol. 3, núm. 4., (2019)

David Ricardo Silva Guachilema; Juan Carlos Salazar; Johnny Correa Michilena; Javier Stalin Villagómez Toral

- Primordialmente, le otorga al especialista tratante la seguridad suficiente para poder dar de alta a un paciente que ha padecido un incidente de dolor en el pecho " $\mathrm{y}$ muestra un resultado normal de Tnc", y;

- Facilita el descarte, de un IAM en un paciente con dolor en el pecho (dolor torácico), casi con absoluta probabilidad.

Ha de tenerse claro que, existe un gran número de enfermedades cardiacas (distintas a la IAM) y extracardiacas que producen daño en el corazón (por mecanismos distintos al del infarto) que igualmente pueden elevar el valos de la Tnc, situación ésta que posiblemente pueda asumirse como un aspecto que genera incertidumbre diagnóstica para IAM cuando no se tiene clara la historia clínica del paciente, no obstante, debe considerarse de manera muy especial que, precisamente, es la compatibilidad entre los síntomas y/o el electrocardiograma del paciente con el elevado nivel de la Tnc en muestras sucesivas lo que inequívocamente determina un IAM. (Sociedad Española de Cardiología, 2018)

\section{Conclusión}

Independientemente de que de la reciente literatura científico académica se evidencie que para el diagnóstico de IAM aún priorice la clínica y la apreciación de los síntomas del paciente, no es menos cierto que el uso de la prueba de troponina como biomarcadores cardíacos es hoy en día un método de diagnóstico ampliamente reconocido en la práctica clínica cardiológica, y ello es muy probablemente a su confiable sensibilidad y especificidad, no obstante, en ningún momento se debe dejar de considerar que el IAM no siempre se manifiesta en el paciente bajo los clásicos 


\section{Importancia de las troponinas en el diagnóstico de infarto agudo de miocardio}

(IAM)

Vol. 3, núm. 4., (2019)

David Ricardo Silva Guachilema; Juan Carlos Salazar; Johnny Correa Michilena; Javier Stalin

Villagómez Toral

síntomas, y es precisamente en ese tipo de escenarios en el que la troponina positiva tiene que interpretarse con fundamento en la historia clínica, a fines de diferenciar si se está en presencia de algún tipo de infarto de miocardio o de una lesión miocárdica aguda, para entonces poder proceder a indicar el tratamiento idóneo.

\section{Biliografía}

A.D.A.M. - Enciclopedia Médica. (17 de Octubre de 2017). Prueba de troponina. Recuperado el 20 de 07 de 2019, de https://medlineplus.gov/spanish/ency/article/007452.htm

Bazzino, Ó. (Diciembre de 2013). Tercera definición universal de infarto de miocardio. Revista Uruguaya de Cardiología, 28(3), 403 - 411. Recuperado el 23 de 07 de 2019, de http://www.scielo.edu.uy/scielo.php?pid=S1688-

04202013000300014\&script=sci_arttext\&tlng=en\#1

Calvachi, P., Barrios, D., Puccini, M., Mojica, H., Delgadillo, D., Gómez, M., . . Buitrago, A. (Noviembre - Deciembre de 2017). Frecuencia de los tipos de infarto agudo de miocardio según la tercera definición. Revista Colombiana de Cardiología, 24(6), 592-597. doi: 10.1016/j.rccar.2017.06.008

Clínica Universidad de Navarra. (2019 a). Clínica Universidad de Navarra. Recuperado el 23 de 07 de 2019, de Análisis diagnóstico del infarto agudo de miocardio en la clínica: https://www.cun.es/enfermedades-tratamientos/pruebas-diagnosticas/diagnostico-infartoagudo-miocardio

Clinica Universidad de Navarra. (2019 b). Clinica Universidad de Navarra. Recuperado el 23 de 07 de 2019, de Diagnóstico y tratamiento del infarto de miocardio en la clínica: https://www.cun.es/enfermedades-tratamientos/enfermedades/infarto-miocardio

Hospital Universitario Austral. (2019). Infarto Agudo de Miocardio. Recuperado el 20 de 07 de 2019, de https://www.hospitalaustral.edu.ar/enfermedades/infarto-agudo-de-miocardio/

Pérez, P. (2019). Infarto de Miocardio. Recuperado el 22 de 07 de 2019, de https://fundaciondelcorazon.com/informacion-para-pacientes/enfermedadescardiovasculares/infarto.html 
Importancia de las troponinas en el diagnóstico de infarto agudo de miocardio (IAM)

Vol. 3, núm. 4., (2019)

David Ricardo Silva Guachilema; Juan Carlos Salazar; Johnny Correa Michilena; Javier Stalin Villagómez Toral

SEMI. (05 de Agosto de 2019). Infarto Agudo de Miocardio. Recuperado el 10 de 07 de 2019, de https://www.fesemi.org/informacion-pacientes/conozca-mejor-su-enfermedad/infartoagudo-de-miocardio

Sociedad Española de Cardiología. (06 de Noviembre de 2018). Sociedad Española de Cardiología. Recuperado el 24 de 07 de 2019, de Documento de consenso sobre la utilización de troponina cardiaca en Urgencias: https://secardiologia.es/comunicacion/notas-de-prensa/notas-de-prensa-sec/10015documento-de-consenso-sobre-la-utilizacion-de-troponina-cardiaca-en-urgencias

$$
\text { (C) (1) } \Theta(0)
$$

RECONOCIMIENTO-NOCOMERCIAL-COMPARTIRIGUAL

CC BY-NC-SA

ESTA LICENCIA PERMITE A OTROS ENTREMEZCLAR, AJUSTAR Y CONSTRUIR A PARTIR DE SU OBRA CON FINES NO COMERCIALES, SIEMPRE Y CUANDO LE RECONOZCAN LA AUTORÍA Y SUS NUEVAS CREACIONES ESTÉN BAJO UNA LICENCIA CON LOS MISMOS TÉRMINOS. 